\title{
Identification of the active compounds and significant pathways of yinchenhao decoction based on network pharmacology
}

\author{
JIHAN HUANG $^{1}$, FAN CHEUNG ${ }^{2}$, HOR-YUE TAN $^{2}$, MING HONG $^{2}$, \\ NING WANG ${ }^{2}$, JUAN YANG ${ }^{1}$, YIBIN FENG ${ }^{2}$ and QINGSHAN ZHENG ${ }^{1}$ \\ ${ }^{1}$ Center for Drug Clinical Research, Shanghai University of Traditional Chinese Medicine, Shanghai 201203; ${ }^{2}$ School \\ of Chinese Medicine, Li Ka Shing Faculty of Medicine, The University of Hong Kong, Hong Kong, SAR, P.R. China
}

Received April 3, 2017; Accepted July 28, 2017

DOI: $10.3892 / \mathrm{mmr} .2017 .7149$

\begin{abstract}
Yinchenhao decoction (YCHD) is a traditional Chinese medicine formulation, which has been widely used for the treatment of jaundice for 2,000 years. Currently, YCHD is used to treat various liver disorders and metabolic diseases, however its chemical/pharmacologic profiles remain to be elucidated. The present study identified the active compounds and significant pathways of YCHD based on network pharmacology. All of the chemical ingredients of YCHD were retrieved from the Traditional Chinese Medicine Systems Pharmacology database. Absorption, distribution, metabolism and excretion screening with oral bioavailability (OB) screening, drug-likeness (DL) and intestinal epithelial permeability (Caco-2) evaluation were applied to discover the bioactive compounds in YCHD. Following this, target prediction, pathway identification and network construction were employed to clarify the mechanism of action of YCHD. Following OB screening, and evaluation of DL and Caco-2, 34 compounds in YCHD were identified as potential active ingredients, of which 30 compounds were associated with 217 protein targets. A total of 31 significant pathways were obtained by performing enrichment analyses of 217 proteins using the JEPETTO 3.x plugin, and 16 classes of gene-associated diseases were revealed by performing enrichment analyses using Database for Annotation, Visualization and Integrated Discovery v6.7. The present study identified potential active compounds and significant pathways in YCHD. In addition, the mechanism of action of YCHD in the treatment of various diseases through multiple pathways was clarified.
\end{abstract}

Correspondence to: Professor Yibin Feng, School of Chinese Medicine, Li Ka Shing Faculty of Medicine, The University of Hong Kong, 10 Sassoon Road, Pokfulam, Hong Kong, SAR, P.R. China E-mail: yfeng@hku.hk

Professor Qingshan Zheng, Center for Drug Clinical Research, Shanghai University of Traditional Chinese Medicine, 1200 Cailun Road, Shanghai 201203, P.R. China

E-mail: qingshan.zheng@drugchina.net

Key words: yinchenhao decoction, network pharmacology, intracellular targets, significant pathways

\section{Introduction}

Yinchenhao decoction (YCHD) is a classical traditional Chinese medicine (TCM) formulation. YCHD has been used widely for the treatment of Yang jaundice and liver disorders. YCHD is composed of three Chinese medicinal herbs: Artemisiae scopariae herba (ASH, Yinchen), Radix et Rhizoma Rhei (RERR, Dahuang) and Gardeniae Fructus (GF, Zhizi). Pharmacologic studies have shown that this formulation can also be used to treat pancreatic carcinoma (1), liver injury $(2,3)$, liver fibrosis (4), liver cirrhosis $(5,6)$, nonalcoholic steatohepatitis (7), cholestasis (8) and diabetes mellitus (DM) (9).

In recent years, TCM monomers and TCM compounds have been studied extensively worldwide. Liu et al (10) predicted the molecular targets of YCHD based on systems-biology methods using the TCMGeneDIT database. However, only 17 main compounds were analyzed, and no active component in GF was identified (11). Other studies have focused only on the molecular mechanism of a certain aspect of YCHD, for example, immunity and metabolism, transport, signal transduction, and cell growth/proliferation (12). Previously, we found that genipin, a single component of GF, had inhibitory effects on human hepatocellular carcinoma cells (13). However, the active substances of YCHD and its specific molecular mechanism of action in the diseases mentioned above are not clear.

Any TCM formulation is a complex system with multiple components, multiple targets, and synergistic interactions among its components (14). Because of its complex chemical composition, it is extremely difficult to study its role in the body as a mixture. The complexity of TCM formulations makes their in-depth study difficult, whereas systems pharmacology provides new ideas and perspectives for the study of Chinese herbal compounds. Studies on the active substances of TCM formulations, identification of the targets of active components, and determination of the relationship between efficacious substances and diseases using systems pharmacology (15) and network pharmacology (16) can help elucidate the molecular mechanism of action of TCM formulations.

For Chinese herbal compounds administered via the oral route, the ingredients in a TCM formulation must first overcome the barriers posed by ADME (absorption, distribution, metabolism and excretion) processes, and only the molecules 
that pass through the barriers may be classed as 'active molecules' (17). These molecules bind to the targets in the body, thereby eliciting their actions. Then, drugs interact with the human body at the network level, as well as the overall level of the organ.

Therefore, based on analyses of ADME-related properties, identification of the active molecules in TCM formulations that pass across the body barrier and prediction of the network targets of active substances was undertaken. Thereafter, studies on the overall effect on the body, as well as the mechanism of action, was carried out. This strategy could provide a basis for in-depth understanding of the mechanism of action of TCM formulations. The workflow of the network-pharmacology approach in the present study is illustrated in Fig. 1.

\section{Materials and methods}

Identification of candidate compounds. All compounds of the three Chinese medicinal herbs in YCHD were collected from the Traditional Chinese Medicine Systems Pharmacology Database and Analysis Platform (TCMSP) database. The TCMSP database consists of 500 Chinese herbal medicines registered in the Chinese Pharmacopoeia (2010 edition) with 30,069 ingredients through literature mining and database integration. Data relevant to the pharmacokinetic properties of each chemical compound, which contained the prediction of oral bioavailability (OB), intestinal epithelial permeability (Caco-2 cells), drug-likeness (DL), blood-brain barrier (BBB), drug half-life (HL) and Lipinski's rule (LR) of five, were provided for the screening and evaluation of compounds (17).

Screening of active compounds. In ADME processes, $\mathrm{OB}$ is one of the most important pharmacokinetic parameters (18). High OB is often a key indicator to determine the DL of bioactive molecules. For TCM formulations, the failure of most of the ingredients to reach the protein target sites of particular cells is due to a lack of appropriate pharmacologic properties, especially $\mathrm{OB}$. Molecules with $\mathrm{OB} \geq 30 \%$ were considered to have good $\mathrm{OB}$ in the present study.

In the early stages of drug development, DL evaluation helps to screen out excellent compounds (19) and increases the 'hit rate' of drug candidates. Therefore, the DL of molecules in YCHD was assessed using the Tanimoto coefficient in the present study (20) using the following formula:

$$
T(X, Y)=\frac{x * y}{x^{2}+y^{2}-x * y}
$$

Where $\mathrm{x}$ is the molecular descriptor of YCHD based on Dragon software (http:www.talete.mi.it/products/dragon_description.htm) and $y$ is the average descriptor of all drugs in the Drugbank database. The average DL Index of all drugs in the Drugbank database is 0.18 , which indicates a high DL. Thus in our study, active molecules were defined as those with a DL Index $\geq 0.18$.

The intestinal epithelial permeability can be investigated using Caco-2 cells (21). Orally administered drugs are absorbed mainly through intestinal epithelial cells. Therefore, simulation of drug transport across the monolayers of small-intestinal epithelial cells is crucial for the prediction of drug absorption. The permeability of epithelial cells of ingredients in Chinese herbal medicines was predicted using the TCMSP database. It was considered that molecules with Caco-2 >-0.40 had good permeability in the small-intestinal epithelium.

Hence, the selected candidate molecules had to meet the requirements of $\mathrm{OB} \geq 30 \%, \mathrm{DL} \geq 0.18$ and Caco- $2>-0.40$ for further analyses.

Identification of associated proteins and gene names. Protein targets were retrieved from the TCMSP database (http://lsp. nwsuaf.edu.cn/tcmsp.php). The dataset used in model-building comprised 6511 drug molecules and 3987 targets for which the compound-protein interactions are known in the Drugbank database (17). UniProt Knowledgebase (UniProtKB) is a protein database containing 54,247,468 sequence entries. The gene names were extracted further from the UniProtKB (http://www.uniprot.org).

Identification of significant pathways and gene-associated diseases. Java Enrichment of Pathways Extended to Topology (JEPETTO) is a Cytoscape 3.x plugin that performs integrative analyses of human gene sets. It can also identify functional associations between genes and known cellular pathways and processes using protein-interaction networks and topologic analyses (22). Significant pathways can be identified by enrichment analyses of proteins using JEPETTO. Analyses of gene-associated diseases were performed with acquired genetic information by the Database for Annotation, Visualization and Integrated Discovery (DAVID) v6.7 (23).

Construction of a network and analyses. The Compound-Target Network was built by connecting the candidate compounds and corresponding targets. The Compound-Pathway Network was generated by linkage of the candidate compounds and the signaling pathways involved. In the Gene-Disease Network, diseases were connected with the associated candidate targets. The corresponding diseases of potential genes were collected by DAVID enrichment analyses, and the obtained interactions between diseases and genes were applied further for building the Gene-Disease Network.

In this bilateral network, the 'nodes' represented the compounds, protein targets, signal pathways or diseases, and 'edges' represented the interactions of Compound-Target, Compound-Pathway or Gene-Disease. The networks were constructed using Cytoscape v3.3.0 (24).

\section{Results}

Identification of the active compounds in YCHD. Using the TCMSP database, 236 compounds were retrieved: 53 in ASH, 92 in RERR, and 98 in GF (3 herbs shared 7 compounds). The network flowchart of the compounds in YCHD is shown in Fig. 1. Of the 53 compounds in ASH, 34 satisfied the criterion of $\mathrm{OB} \geq 30 \%$, and 13 satisfied the criteria of $\mathrm{OB} \geq 30 \%, \mathrm{DL} \geq 0.18$ and Caco- $2 \geq-0.4$. Of the 92 compounds in RERR, 26 satisfied the criterion of $\mathrm{OB} \geq 30 \%, 16$ satisfied the criteria of $\mathrm{OB} \geq 30 \%$ and DL $\geq 0.18$, and 9 satisfied the criteria of $\mathrm{OB} \geq 30 \%$, DL $\geq 0.18$ and Caco- $2 \geq-0.4$. Of the 98 compounds in GF, 43 satisfied the criterion of $\mathrm{OB} \geq 30 \%$, and 15 satisfied the criteria of $\mathrm{OB} \geq 30 \%$ and $\mathrm{DL} \geq 0.18$, and 14 satisfied the criteria of OB 
Table I. The number of compounds in YCHD satisfy $\mathrm{OB} \geq$ $30 \%, \mathrm{DL} \geq 0.18$ and Caco- $2 \geq-0.4$

\begin{tabular}{lcccc}
\hline Herbs & Total & OB $\geq 30 \%$ & DL $\geq 0.18$ & Caco- $\geq-0.4$ \\
\hline ASH & 53 & $34(64.2)$ & $13(24.5)$ & $13(24.5)$ \\
RRER & 92 & $26(28.3)$ & $16(17.4)$ & $9(9.8)$ \\
GF & 98 & $43(48.9)$ & $15(15.3)$ & $14(14.3)$ \\
\hline
\end{tabular}

YCHD, yinchenhao decoction; OB, oral bioavailability; DL, drug-likeness.

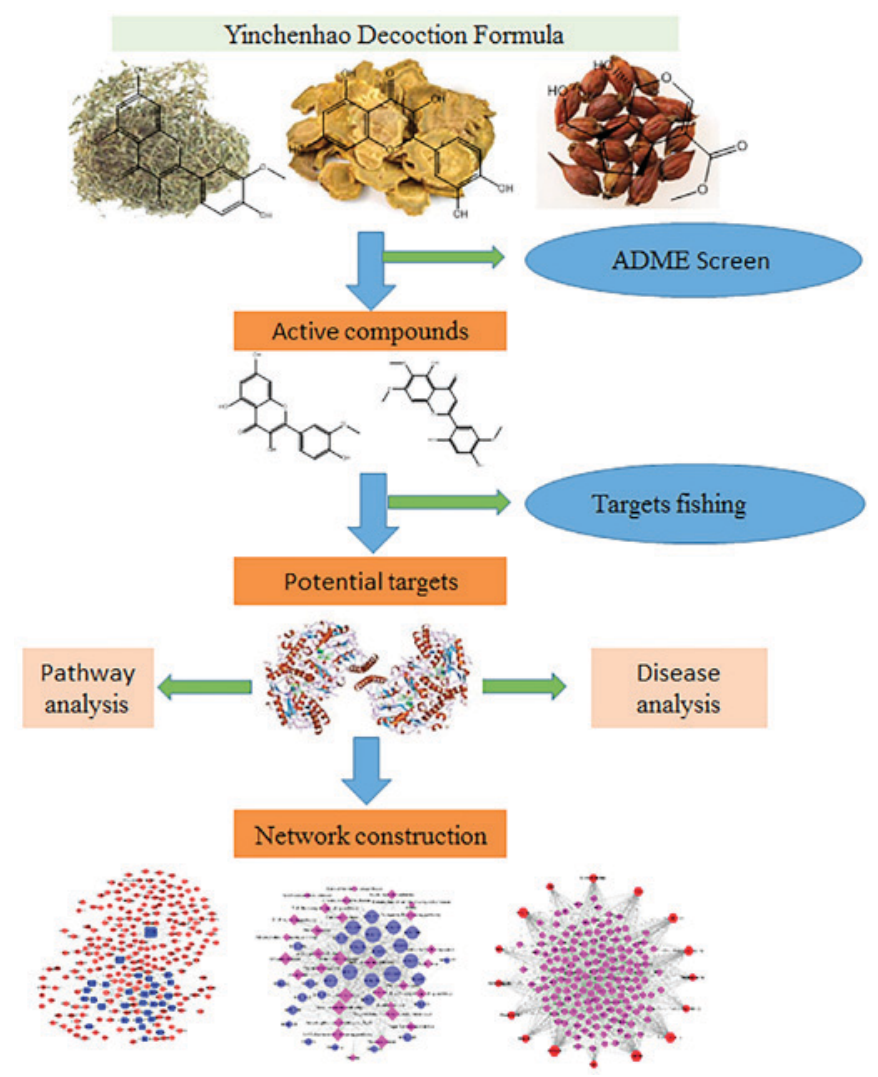

Figure 1. The workflow for the network-pharmacology approach used in our study.

$\geq 30 \%$, DL $\geq 0.18$ and Caco- $2 \geq-0.4$. Among 243 compounds, 36 compounds satisfied all of the pre-defined requirements (Table I) and, finally, 33 compounds were analyzed after removing duplicates. The OB of genipin was $<30 \%$, but it was a common compound in GF and was shown to have inhibitory effects on human hepatocellular carcinoma cells in our previous study (13). Hence, genipin was also regarded to be a candidate compound. The details of 34 compounds are shown in Table II. Interestingly, all three Chinese medicinal herbs in YCHD (i.e., ASH, RERR and GF) contained beta-sitosterol, whereas RERR and GF contained quercetin. Beta-sitosterol and quercetin are the common chemicals found in $188 \mathrm{TCM}$ formulations according to the TCMSP database.

Identification of targets in YCHD. Among the 34 compounds obtained, 618 proteins and genes were obtained for
30 compounds, and 217 proteins and genes were included after removing duplicates. The 30 candidate compounds and all of the potential targets were applied to produce a plot of Compound-Target interactions, including 247 nodes (30 compounds and 217 targets) and 618 edges (Fig. 2). In Fig. 2, the red nodes are drug targets and the blue nodes are compounds, and the edges represent the interactions between them. The centralization and heterogeneity of the network was 0.603 and 2.355 , respectively. This finding indicated that some nodes were more concentrated in the network than others. That is, the Compound-Target space was biased towards certain compounds and targets. As depicted in Fig. 2, MOL053 (quercetin) displayed the most target interactions (degree=154), followed by MOL173 (kaempferol, degree=63), MOL015 (beta-sitosterol, degree=38), MOL014 (isorhamnetin, degree=37) and MOL174 (stigmasterol, degree=31).

A TCM formulation is a complex system with various components; one component may act on multiple targets and display synergistic effects to treat diseases. These compounds with high degree nodes may perform important roles in the pharmacologic effect of YCHD. Protein targets acting as 'hubs' in the network were prostaglandin $\mathrm{G} / \mathrm{H}$ synthase 2 (PTGS2; 28 interactions), heat-shock protein HSP 90 (20 interactions), prostaglandin $\mathrm{G} / \mathrm{H}$ synthase 1 (19 interactions), nuclear receptor coactivator 2 (18 interactions), dipeptidyl peptidase IV (18 interactions) and mRNA of PKA catalytic subunit $\mathrm{C}$-alpha (17 interactions).

Revealing the significant pathways. Enrichment analyses of 217 proteins were done using JEPETTO and 31 significant pathways were obtained (Table III). This XD-score is relative to the average distance to all pathways and represents a deviation (positive or negative) from the average distance. The q-value determines the significance of the overlap (Fisher's exact test) between the input information and the pathways. The Overlap/Size shows the number of overlapping proteins compared with the size of the pathway.

Enrichment algorithm analyses of the XD-score and q-value revealed the highest XD-score to be 1.47032, and the threshold value of XD-score in our study was 0.35 . Eighteen disease pathways included 11 cancer pathways (non-small-cell lung cancer, small-cell lung cancer, bladder cancer, prostate cancer, endometrial cancer, colorectal cancer, glioma, pancreatic cancer, chronic myeloid leukemia, acute myeloid leukemia, melanoma) and one immune system-disease pathway (graft-vs.-host disease), three infectious disease-related pathways (leishmaniasis, malaria, Chagas disease), two neurodegenerative-disease pathways (prion diseases, amyotrophic lateral sclerosis) and one metabolic-disease pathway (type-II DM). Thirteen signaling pathways included four pathways involved in the immune system (NOD-like receptor, Toll-like receptor, Fc epsilon RI, B cell receptor), two signal-transduction pathways (ErbB, vascular endothelial growth factor (VEGF) ), two cell growth and death pathways (p53 signaling pathway, apoptosis), three endocrine-system pathways (progesterone-mediated oocyte maturation, gonadotropin-releasing hormone (GnRH) signaling pathway, adipocytokine signaling pathway) and one lipid-metabolism pathway (biosynthesis of steroid hormone). The Compound-Pathway network was constructed with 29 candidate compounds and their significant pathways 
Table II. Information for candidate active compounds from ASH, RRER and GF herbs.

\begin{tabular}{|c|c|c|c|c|c|c|}
\hline Number & Molecule name & $\mathrm{OB}(\%)$ & Caco-2 & DL & Molecular structure & Herb \\
\hline MOL014 & Isorhamnetin & 49.6 & 0.31 & 0.31 & & $\mathrm{ASH}$ \\
\hline MOL015 & Beta-sitosterol & 36.91 & 1.32 & 0.75 & & ASH/RRER/GF \\
\hline MOL020 & Areapillin & 48.96 & 0.6 & 0.41 & & ASH \\
\hline MOL024 & Genkwanin & 37.13 & 0.63 & 0.24 & & ASH \\
\hline MOL028 & Skrofulein & 30.35 & 0.72 & 0.3 & & $\mathrm{ASH}$ \\
\hline MOL030 & Isoarcapillin & 57.4 & 0.4 & 0.41 & & $\mathrm{ASH}$ \\
\hline MOL031 & Eupalitin & 46.11 & 0.62 & 0.33 & & ASH \\
\hline MOL032 & Eupatolitin & 42.55 & 0.16 & 0.37 & & ASH \\
\hline MOL034 & Capillarisin & 57.56 & 0.49 & 0.31 & & ASH \\
\hline MOL036 & 4'-Methylcapillarisin & 72.18 & 0.57 & 0.35 & & $\mathrm{ASH}$ \\
\hline MOL037 & Demethoxycapillarisin & 52.33 & 0.31 & 0.25 & & $\mathrm{ASH}$ \\
\hline MOL038 & Artepillin A & 68.32 & 0.45 & 0.24 & & ASH \\
\hline MOL053 & Quercetin & 46.43 & 0.05 & 0.28 & & $\mathrm{ASH} / \mathrm{GF}$ \\
\hline MOL065 & Eupatin & 50.8 & 0.53 & 0.41 & & RRER \\
\hline MOL081 & Mutatochrome & 48.64 & 1.97 & 0.61 & & RRER \\
\hline MOL098 & Rhein & 47.07 & -0.2 & 0.28 & & RRER \\
\hline MOL111 & Toralactone & 46.46 & 0.86 & 0.24 & & RRER \\
\hline
\end{tabular}


Table II. Continued.

\begin{tabular}{|c|c|c|c|c|c|c|}
\hline Number & Molecule name & $\mathrm{OB}(\%)$ & Caco-2 & $\mathrm{DL}$ & Molecular structure & Herb \\
\hline MOL127 & Daucosterol_qt & 35.89 & 1.35 & 0.7 & & RRER \\
\hline MOL133 & Palmidin A & 32.45 & -0.36 & 0.65 & & RRER \\
\hline MOL138 & Aloe-emodin & 83.38 & -0.12 & 0.24 & & RRER \\
\hline MOL143 & (-)-catechin & 49.68 & -0.03 & 0.24 & & RRER \\
\hline MOL144 & Crocetin & 35.3 & 0.54 & 0.26 & & GF \\
\hline MOL145 & Genipin & 26.06 & -0.37 & 0.10 & & GF \\
\hline MOL146 & $\begin{array}{l}(4 \mathrm{aS}, 6 \mathrm{aR}, 6 \mathrm{aS}, 6 \mathrm{bR}, \\
\text { 8aR,10R,12aR, } \\
\text { 14bS)-10-hydroxy } \\
\text {-2,2,6a,6b,9,9, } \\
\text { 12a-heptamethyl-1,3, } \\
\text { 4,5,6,6a,7,8,8a,10,11, } \\
\text { 12,13,14b- } \\
\text { tetradecahydropicene- } \\
\text { 4a-carboxylic acid }\end{array}$ & 32.03 & 0.61 & 0.76 & & GF \\
\hline MOL147 & Ammidin & 34.55 & 1.13 & 0.22 & & GF \\
\hline MOL156 & Sudan III & 84.07 & 0.42 & 0.59 & & GF \\
\hline MOL173 & Kaempferol & 41.88 & 0.26 & 0.24 & & GF \\
\hline MOL174 & Stigmasterol & 43.83 & 1.44 & 0.76 & & GF \\
\hline MOL196 & Mandenol & 42 & 1.46 & 0.19 & & GF \\
\hline MOL198 & Supraene & 33.55 & 2.08 & 0.42 & & GF \\
\hline MOL212 & Isoimperatorin & 45.46 & 0.97 & 0.23 & & GF \\
\hline MOL216 & Ethyl oleate (NF) & 32.4 & 1.4 & 0.19 & & GF \\
\hline MOL217 & $\begin{array}{l}\text { 5-hydroxy-7-methoxy-2- } \\
\text { (3,4,5-trimethoxyphenyl) } \\
\text { chromone }\end{array}$ & 51.96 & 0.88 & 0.41 & & GF \\
\hline MOL229 & 3-Methylkempferol & 60.16 & 0.37 & 0.26 & & GF \\
\hline
\end{tabular}




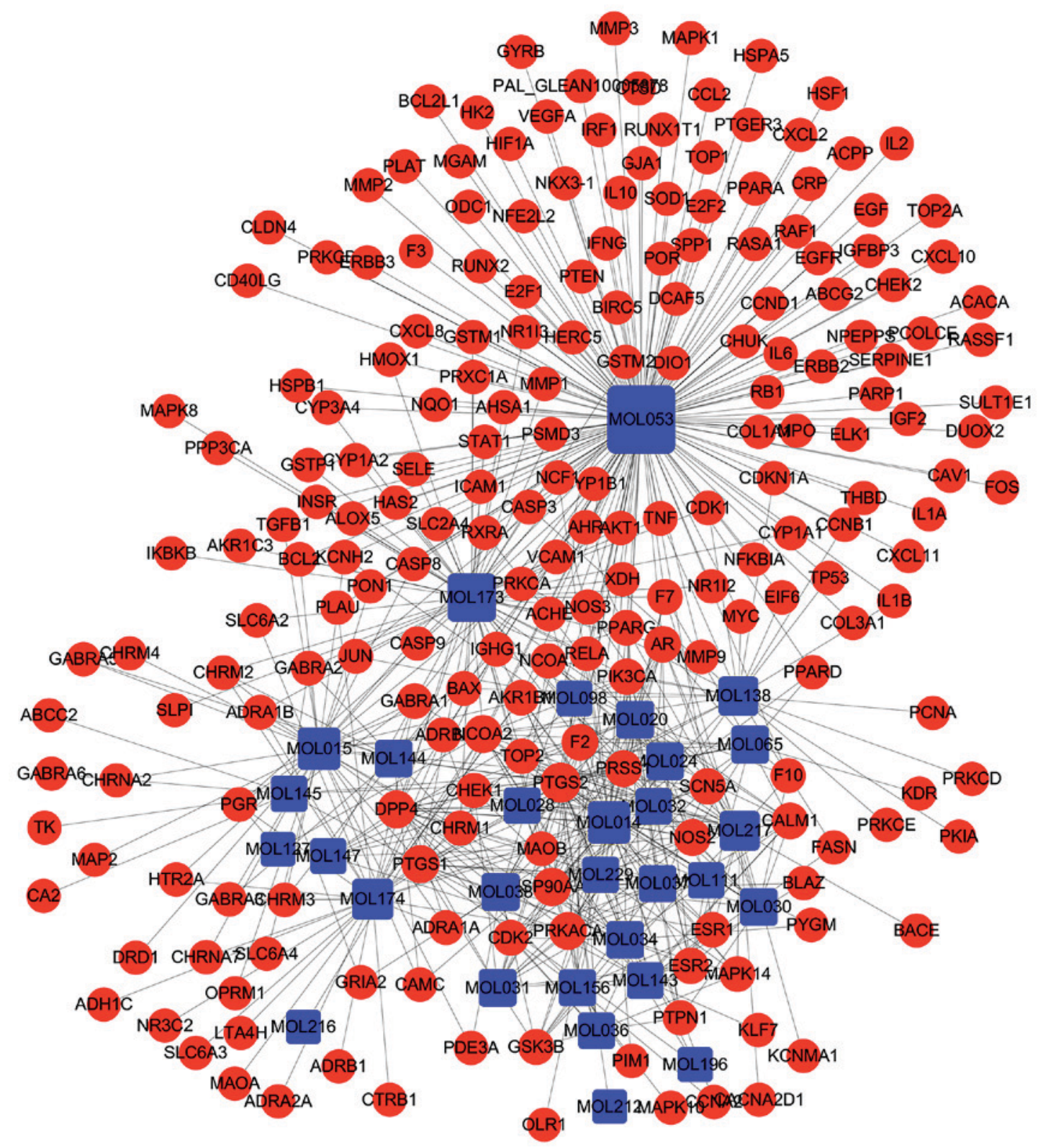

Figure 2. Compound-Target network for YCHD. The red nodes represent potential drug targets and the blue nodes represent active compounds. The edges represent the interaction between them and the node size is proportional to the degree.

included 60 nodes (29 compounds and 31 pathways) and 468 edges (Fig. 3). Pathways are represented by pink nodes, compounds are represented by blue nodes, and the interactions between them are represented by edges in Fig. 3. Centralization and heterogeneity of the network was 0.273 and 0.533 , respectively. It was found that the VEGF signaling pathway (degree $=28$ ) was linked with 28 chemical molecules, and that other pathways interacted with at least two molecules.

Revealing gene-associated diseases. A Gene-Disease network was constructed to identify the potential targets of diseases in which different compounds act upon (Fig. 4). In the present study, 160 potential targets were found which were associated with 16 classes of diseases. Ninety-six genes were related to cancer, 87 genes were related to metabolism, 81 genes were related to cardiovascular diseases, and 81 genes were related to the immune system. Among 160 genes, there were 42 common targets in four diseases: Cancer, metabolic, cardiovascular and immune. The 42 common targets genes were ADH1C, ADRB2, AR, CCL2, COL1A1, CRP, CYP1A1, CYP1A2, CYP3A4, EGF, ESR1, ESR2, F2, GSTM1, GSTP1, HMOX1, ICAM1, IFNG, IGF2, IL10, IL1A, IL1B, IL6, INSR, MMP1, MMP2, MMP9, MPO, NOS2, NOS3, PLAU, PON1, PPARG, PTGS2, SELE, SERPINE1, SLC6A4, SPP1, TGFB1, TNF, TP53, and VEGFA. Among 160 genes, GSTM1, NOS3, and TNF were common genes of 16 diseases.

Taken together, these results indicate that YCHD can regulate whole-body systems through a complex genes-interaction network, resulting in a certain effect in various diseases.

\section{Discussion}

TCM formulations are composed of multiple ingredients. Their mechanism of action is complex, and may be associated 
Table III. The 31 significant pathways found by JEPETTO.

\begin{tabular}{|c|c|c|c|c|}
\hline Number & Pathway & XD-score & q-value & Overlap/size \\
\hline 1 & Bladder cancer & 1.47032 & 0.00000 & $16 / 38$ \\
\hline 2 & Non-small cell lung cancer & 1.08065 & 0.00000 & $16 / 51$ \\
\hline 3 & Prostate cancer & 1.02555 & 0.00000 & $26 / 84$ \\
\hline 4 & Pancreatic cancer & 1.01321 & 0.00000 & $22 / 70$ \\
\hline 5 & Endometrial cancer & 0.94804 & 0.00000 & $14 / 50$ \\
\hline 6 & Colorectal cancer & 0.79509 & 0.00000 & $18 / 61$ \\
\hline 7 & Metabolism of xenobiotics by cytochrome P450 & 0.77843 & 0.00045 & $6 / 20$ \\
\hline 8 & Glioma & 0.76675 & 0.00000 & $16 / 60$ \\
\hline 9 & Leishmaniasis & 0.6752 & 0.00000 & $17 / 62$ \\
\hline 10 & Steroid hormone biosynthesis & 0.64509 & 0.00931 & $4 / 15$ \\
\hline 11 & Type II diabetes mellitus & 0.62494 & 0.00003 & $10 / 43$ \\
\hline 12 & NOD-like receptor signaling pathway & 0.61233 & 0.00000 & $14 / 59$ \\
\hline 13 & Prion diseases & 0.607 & 0.00004 & $9 / 35$ \\
\hline 14 & Small cell lung cancer & 0.57843 & 0.00000 & $20 / 82$ \\
\hline 15 & ErbB signaling pathway & 0.56917 & 0.00000 & $17 / 84$ \\
\hline 16 & VEGF signaling pathway & 0.56409 & 0.00000 & $14 / 62$ \\
\hline 17 & Chronic myeloid leukemia & 0.53594 & 0.00000 & $16 / 69$ \\
\hline 18 & Malaria & 0.53081 & 0.00003 & $10 / 42$ \\
\hline 19 & Acute myeloid leukemia & 0.52287 & 0.00003 & $11 / 52$ \\
\hline 20 & Chagas disease & 0.51782 & 0.00000 & $22 / 99$ \\
\hline 21 & p53 signaling pathway & 0.49646 & 0.00000 & $15 / 62$ \\
\hline 22 & Toll-like receptor signaling pathway & 0.47843 & 0.00000 & $19 / 90$ \\
\hline 23 & Melanoma & 0.46367 & 0.00003 & $12 / 62$ \\
\hline 24 & Apoptosis & 0.44093 & 0.00000 & $17 / 81$ \\
\hline 25 & Progesterone-mediated oocyte maturation & 0.41387 & 0.00002 & $14 / 79$ \\
\hline 26 & Fc epsilon RI signaling pathway & 0.41091 & 0.00023 & $11 / 65$ \\
\hline 27 & B cell receptor signaling pathway & 0.40613 & 0.00002 & $13 / 69$ \\
\hline 28 & Graft-versus-host disease & 0.37843 & 0.01012 & $5 / 25$ \\
\hline 29 & GnRH signaling pathway & 0.37629 & 0.00003 & $14 / 83$ \\
\hline 30 & Adipocytokine signaling pathway & 0.3679 & 0.00036 & $10 / 57$ \\
\hline 31 & Amyotrophic lateral sclerosis (ALS) & 0.36104 & 0.00007 & $10 / 47$ \\
\hline
\end{tabular}

with multiple targets and multiple pathways. YCHD is a classic TCM formulation and used commonly to treat liver diseases by clearing heat, eliminating dampness, and removing jaundice.

In the present study, we proposed a network pharmacologic approach to identify bioactive compounds and significant pathways in YCHD by OB screening, as well as evaluation of DL and intestinal absorption. Finally, 243 compounds in YCHD were extracted from the TCMSP database, and 33 compounds with good OB, DL and small-intestinal absorption were considered to be active molecules in YCHD for further study.

Some of the compounds have been shown to possess pharmacologic activities for the treatment of various diseases. These include the anti-lung-cancer activity of isorhamnetin (25), as well as beta-sitosterol (analgesic) (26), genkwanin (anti-colorectal cancer) (27), eupalitin (anti-prostate carcinoma) (28), capillarisin (anti-hyperalgesic and anti-allodynic) (29), quercetin (anticancer) (30), rhein (anticancer) (31), aloe-emodin (anti-growth disorders) (32), crocetin (anticancer) (33), Sudan III (anti-persistent chylous ascites) (34), kaempferol (anti-pancreatic cancer) (35), stigmasterol (anticancer) (36). In our study, based on the potential targets that 34 compounds act upon, 31 significant pathways and 16 classes of diseases that were associated with the targets were obtained.

By analyzing the topologic properties of the Compound-Target interaction network, we found that compounds with high degree nodes and protein targets that occupied hub positions in the network could perform important roles in the pharmacologic function of YCHD. Analyses of the Compound-Pathway network showed that the main active ingredients in YCHD could act on multiple pathways, and that the TCM formulations had multiple components, multiple targets and integrated regulation (14). Then, we linked potential genes to diseases, and found that these potential genes were related to several complex diseases: Cancer, cardiovascular, metabolic, and immune. For example, the development and progression of tumors is associated with multiple pathways $(37,38)$. The main advantage of TCM therapeutics is that the many compounds within them exert a more robust 


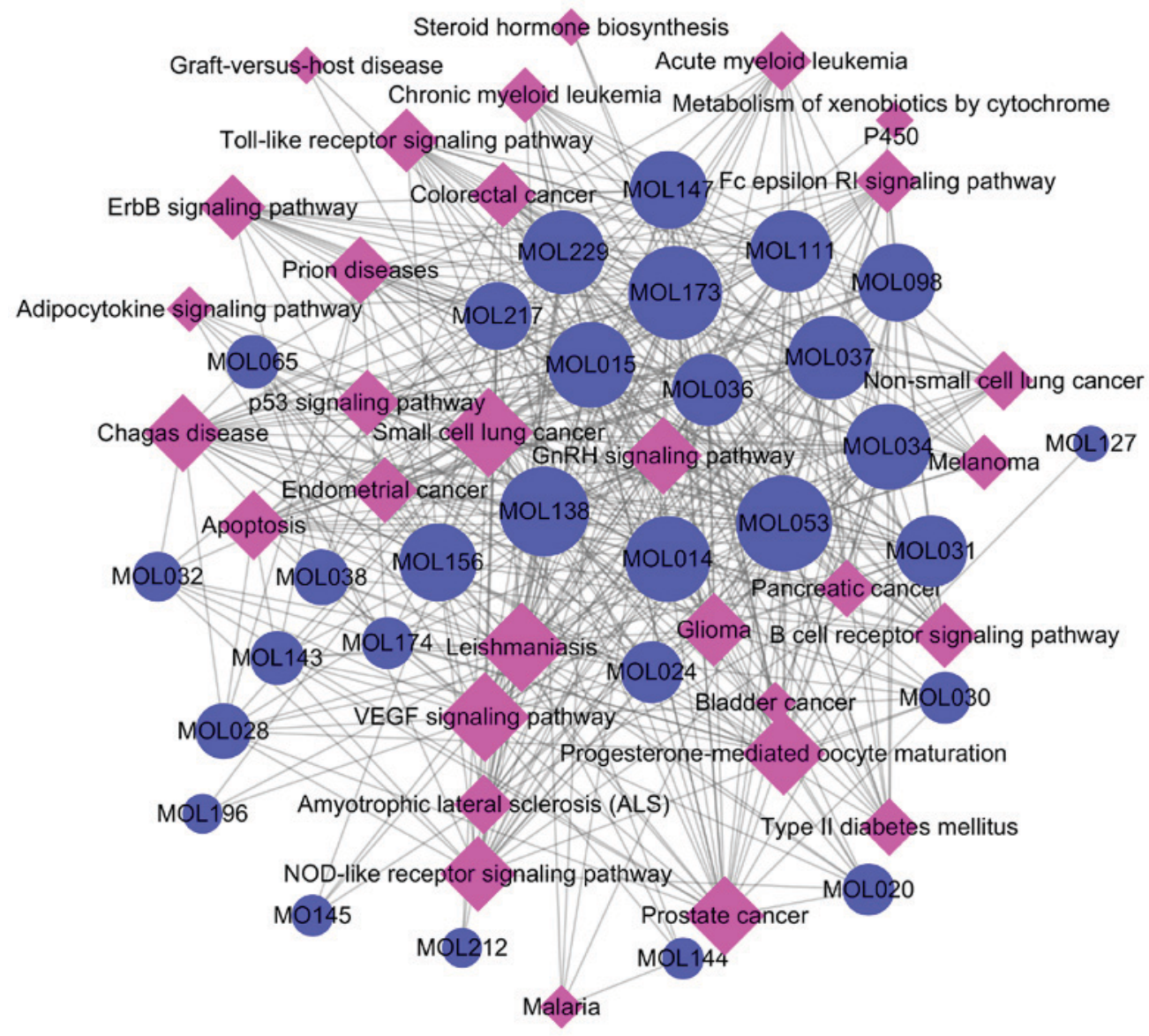

Figure 3. Compound-Pathway network for YCHD. The pink nodes represent significant pathways and the blue nodes represent active compounds. The edges represent the interaction between them and node size is proportional to the degree.

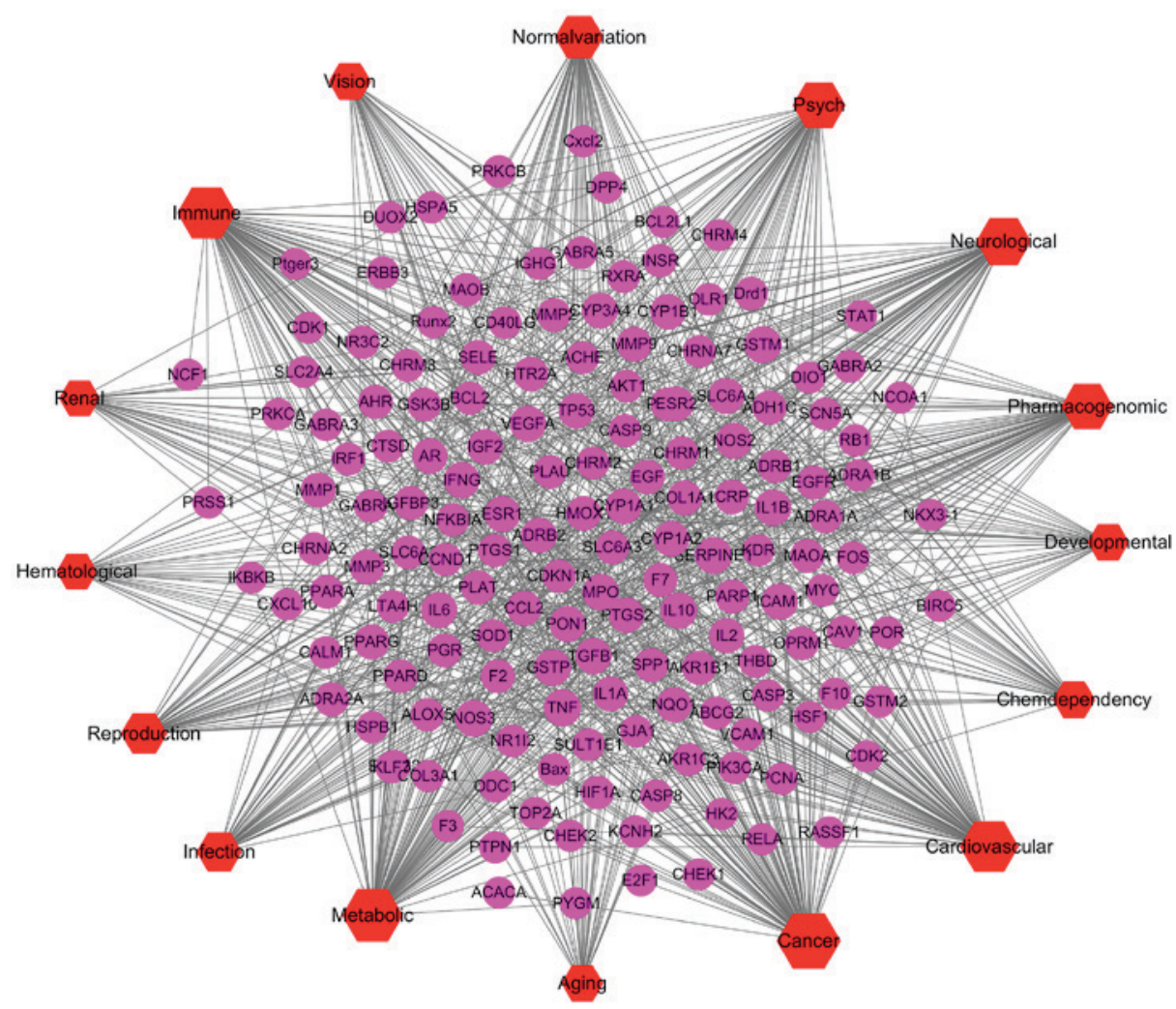

Figure 4. Gene-Disease network for YCHD. The red nodes represent disease and the pink nodes represent genes. The edges represent the interaction between them and node size is proportional to the degree. 
synergistic effect than any individual compound by 'hitting' multiple targets. Therefore, the multidirectional mechanisms of TCM formulations that act through multiple pathways of the immune system, endocrine system, signal transduction and cell growth and death may offer a new therapeutic tool to treat tumors.

Studies have shown that the active molecules in YCHD include beta-sitosterol (39), isorhamnetin (25), genkwanin (27), eupalitin (28) and quercetin (30) and have anticancer effects. In particular, quercetin is a potent antioxidant flavonoid found in many common medicinal herbs, and possesses a wide spectrum of biologic activities (40). Quercetin may have cardioprotective, anticancer, anti-ulcer, anti-allergic, anti-viral, anti-inflammatory, anti-DM, gastroprotective, anti-hypertensive, anti-infective and immunomodulatory activities (41). Our network study also showed that YCHD can act on cancer, metabolic, cardiovascular, and immune systems. Disease enrichment analyses showed that 96 genes were related to cancer. Pathway enrichment analyses demonstrated YCHD to be involved with regulation of multiple pathways in cancer, including apoptosis (1) as well as various signaling pathways: NOD-like receptor (42), Toll-like receptor (43), Fc epsilon RI (44), B-cell receptor (45), ErbB (46), VEGF (47), and GnRH (48). Therefore, YCHD may exert anticancer effects through regulation of cell death, anti-inflammation, anti-immune system, anti-angiogenesis, and energy metabolism (37). Our study also verified a report stating that YCHD has pharmacologic activities against primary liver cancer (49), pancreatic carcinoma (1), and DM (9).

In most cases, the occurrence and development of a disease can also be considered to be a result of a network $(50,51)$. Interestingly, we found 42 common targets in four diseases (cancer, metabolic, cardiovascular, and immune) among 160 genes. We also showed that a disease does not occur in isolation. Studies using a one-target and one-drug model tend to ignore the relationship between diseases (52). The constituents of TCM formulations are complex, and the effect that a single component produces may be relatively weak. However, these ingredients with different effects and different targets can act on various aspects of the disease through systems, and they interact with each other to produce synergistic effects $(53,54)$. Network pharmacology can be used to predict the target profiles and pharmacologic actions of herbal compounds. In our study, network-construction approaches were applied to identify bioactive compounds and potential targets and to determine the underlying mechanism of action of YCHD. However, additional experiments must be carried out to validate our study results.

Thirty-four bioactive compounds with 31 significant pathways in YCHD were identified by performing network analyses, which explains how to treat disease through multiple components, targets and pathways. The method of network pharmacology developed in our study could provide novel insights into the mechanism of action of YCHD.

\section{Acknowledgements}

The present study was supported by the Shanghai Education Commission (ZY3-CCCX-3-1001), the Research Council of the University of Hong Kong (104003422, 104004092) and the Research Grants Committee of Hong Kong, HKSAR (17152116).

\section{References}

1. Zhou HB, Chen JM, Shao LM and Chen ZG: Apoptosis of human pancreatic carcinoma cell-1 cells induced by Yin Chen Hao Decoction. World J Gastroenterol 21: 8352-8357, 2015.

2. Jiang SL, Hu XD and Liu P: Immunomodulation and liver protection of Yinchenhao decoction against concanavalin A-induced chronic liver injury in mice. J Integr Med 13: 262-268, 2015.

3. Cao HX, Sun H, Jiang XG, Lu HT, Zhang GM, Wang XJ, Sun WJ, Wu ZM, Wang P, Liu L and Zhou J: Comparative study on the protective effects of Yinchenhao Decoction against liver injury induced by alpha-naphthylisothiocyanate and carbon tetrachloride. Chin J Integr Med 15: 204-209, 2009.

4. Wang YH, Zhao CX, Chen BM, He M, Liu LQ, Li CY and Chen X: Reverse effect of Yinchenhao decoction in dimethyl nitrosamine-induced hepatic fibrosis in rats. Zhongguo Zhong Yao Za Zhi 39: 1473-1478, 2014 (In Chinese).

5. Liu C, Liu P and Tao Q: Recipe-syndrome correlation and pathogenesis mechanism of Yinchenhao Decoction in intervening dimethylnitrosamine induced liver cirrhosis progress in rats. Zhongguo Zhong Xi Yi Jie He Za Zhi 30: 845-850, 2010 (In Chinese).

6. Sun MY, Wang L, Mu YP, Liu C, Bian YQ, Wang XN and Liu P: Effects of Chinese herbal medicine Yinchenhao Decoction on expressions of apoptosis-related genes in dimethylnitrosamineor carbon tetrachloride-induced liver cirrhosis in rats. Zhong Xi Yi Jie He Xue Bao 9: 423-434, 2011 (In Chinese).

7. Chen SD, Fan Y and Xu WJ: Effects of yinchenhao decoction (see text) for non-alcoholic steatohepatitis in rats and study of the mechanism. J Tradit Chin Med 31: 220-223, 2011.

8. Chen Z, Ma X, Zhao Y, Wang J, Zhang Y, Li J, Wang R, Zhu Y, Wang $\mathrm{L}$ and Xiao $\mathrm{X}$ : Yinchenhao decoction in the treatment of cholestasis: A systematic review and meta-analysis. J Ethnopharmacol 168: 208-216, 2015.

9. Pan J, Han C, Liu H, Du J and Li A: Effects of yinchenhao decoction on normal animals and animal models of diabetes mellitus. Zhong Yao Cai 24: 128-131, 2001 (In Chinese).

10. Liu T, Huang HB, Lin ZC, Liu Q and Zhu W: Exploring the potential molecular target proteins of yinchenhao decoction using computer systemic biology. Zhong Yao Cai 34: 1648-1651, 2011 (In Chinese).

11. Fang YC, Huang HC, Chen HH and Juan HF: TCMGeneDIT: A database for associated traditional Chinese medicine, gene and disease information using text mining. BMC Complement Altern Med 8: 58, 2008

12. Zhang A, Sun H, Qiu S and Wang X: Advancing drug discovery and development from active constituents of yinchenhao tang, a famous traditional chinese medicine formula. Evid Based Complement Alternat Med 2013: 257909, 2013.

13. Wang N, Zhu M, Tsao SW, Man K, Zhang Z and Feng Y: Up-regulation of TIMP-1 by genipin inhibits MMP-2 activities and suppresses the metastatic potential of human hepatocellular carcinoma. PloS One 7: e46318, 2012.

14. Ma YM, Zhang XZ, Su ZZ, Li N, Cao L, Ding G, Wang ZZ and Xiao W: Insight into the molecular mechanism of a herbal injection by integrating network pharmacology and in vitro. J Ethnopharmacol 173: 91-99, 2015.

15. Berger SI, Ma'ayan A and Iyengar R: Systems pharmacology of arrhythmias. Sci Signal 3: ra30, 2010.

16. Hopkins AL: Network pharmacology. Nat Biotechnol 25: 1110-1111, 2007.

17. Ru J, Li P, Wang J, Zhou W, Li B, Huang C, Li P, Guo Z, Tao W, Yang Y, et al: TCMSP: A database of systems pharmacology for drug discovery from herbal medicines. J Cheminform 6: 13 , 2014.

18. Xu X, Zhang W, Huang C, Li Y, Yu H, Wang Y, Duan J and Ling Y: A novel chemometric method for the prediction of human oral bioavailability. Int J Mol Sci 13: 6964-6982, 2012.

19. Tao W, Xu X, Wang X, Li B, Wang Y, Li Y and Yang L: Network pharmacology-based prediction of the active ingredients and potential targets of Chinese herbal Radix Curcumae formula for application to cardiovascular disease. J Ethnopharmacol 145: $1-10,2013$.

20. Yamanishi Y, Kotera M, Kanehisa M and Goto S: Drug-target interaction prediction from chemical, genomic and pharmacological data in an integrated framework. Bioinformatics 26: i2 $46-\mathrm{i} 254,2010$. 
21. Huang C, Zheng C, Li Y, Wang Y, Lu A and Yang L: Systems pharmacology in drug discovery and therapeutic insight for herbal medicines. Brief Bioinform 15: 710-733, 2014

22. Winterhalter C, Widera P and Krasnogor N: JEPETTO: A Cytoscape plugin for gene set enrichment and topological analysis based on interaction networks. Bioinformatics 30: 1029-1030, 2014.

23. Huang da W, Sherman BT and Lempicki RA: Systematic and integrative analysis of large gene lists using DAVID bioinformatics resources. Nat Protoc 4: 44-57, 2009.

24. Shannon P, Markiel A, Ozier O, Baliga NS, Wang JT, Ramage D, Amin N, Schwikowski B and Ideker T: Cytoscape: A software environment for integrated models of biomolecular interaction networks. Genome Res 13: 2498-2504, 2003.

25. Li Q, Ren FQ, Yang CL, Zhou LM, Liu YY, Xiao J, Zhu L and Wang ZG: Anti-proliferation effects of isorhamnetin on lung cancer cells in vitro and in vivo. Asian Pac J Cancer Prev 16 3035-3042, 2015.

26. Villasenor IM, Angelada J, Canlas AP and Echegoyen D: Bioactivity studies on beta-sitosterol and its glucoside. Phytother Res 16: 417-421, 2002.

27. Wang X, Song ZJ, He X, Zhang RQ, Zhang CF, Li F, Wang CZ and Yuan CS: Antitumor and immunomodulatory activity of genkwanin on colorectal cancer in the APC(Min/+) mice. Int Immunopharmacol 29: 701-707, 2015.

28. Kaleem S, Siddiqui S, Siddiqui HH, Badruddeen, Hussain A, Arshad M, Akhtar J and Rizvi A: Eupalitin induces apoptosis in prostate carcinoma cells through ROS generation and increase of caspase-3 activity. Cell Biol Int 40: 196-203, 2016.

29. Khan S, Shehzad O, Chun J, Choi RJ, Park S, Islam MN, Choi JS and Kim YS: Anti-hyperalgesic and anti-allodynic activities of capillarisin via suppression of inflammatory signaling in animal model. J Ethnopharmacol 152: 478-486, 2014.

30. Kashyap D, Mittal S, Sak K, Singhal P and Tuli HS: Molecular mechanisms of action of quercetin in cancer: Recent advances. Tumour Biol 37: 12927-12939, 2016.

31. Wu C, Cao H, Zhou H, Sun L, Xue J, Li J, Bian Y, Sun R, Dong S, Liu P and Sun M: Research progress on the antitumor effects of rhein: Literature review. Anticancer Agents Med Chem: Sep 30 2015 (Epub ahead of print).

32. Yang M, Li L, Heo SM and Soh Y: Aloe-emodin induces chondrogenic differentiation of ATDC5 cells via MAP kinases and BMP-2 signaling pathways. Biomol Ther (Seoul) 24: 395-401, 2016.

33. Gutheil WG, Reed G, Ray A, Anant S and Dhar A: Crocetin: An agent derived from saffron for prevention and therapy for cancer. Curr Pharm Biotechnol 13: 173-179, 2012.

34. Spagnol L, Conforti A, Valfrè L, Morini F and Bagolan P. Preoperative administration of Sudan III and successful treatment of persistent chylous ascites in a neonate. J Pediatr Surg 46: 994-997, 2011.

35. Lee J and Kim JH: Kaempferol inhibits pancreatic cancer cell growth and migration through the blockade of EGFR-related pathway in vitro. PloS One 11: e0155264, 2016.

36. Ali H, Dixit S, Ali D, Alqahtani SM, Alkahtani S and Alarifi S: Isolation and evaluation of anticancer efficacy of stigmasterol in a mouse model of DMBA-induced skin carcinoma. Drug Des Devel Ther 9: 2793-2800, 2015.
37. Hanahan D and Weinberg RA: Hallmarks of cancer: The next generation. Cell 144: 646-674, 2011

38. Hanahan D and Weinberg RA: The hallmarks of cancer. Cell 100 57-70, 2000.

39. Bin Sayeed MS and Ameen SS: Beta-Sitosterol: A promising but orphan nutraceutical to fight against cancer. Nutr Cancer 67: 1214-1220, 2015.

40. Anand David AV, Arulmoli R and Parasuraman S: Overviews of biological importance of quercetin: A bioactive flavonoid. Pharmacogn Rev 10: 84-89, 2016.

41. Lakhanpal P and Rai D: Quercetin: A versatile flavonoid. Int J Med Update 2: 22-37, 2007.

42. Miskiewicz A, Szparecki G, Durlik M,Rydzewska G,ZiobrowskiI and Gorska R: The Q705K and F359L single-nucleotide polymorphisms of NOD-like receptor signaling pathway: Association with chronic pancreatitis, pancreatic, cancer and periodontitis. Arch Immunol Ther Exp (Warsz) 63: 485-494, 2015.

43. Ntoufa S, Vilia MG, Stamatopoulos K, Ghia P and Muzio M Toll-like receptors signaling: A complex network for NF- $\mathrm{kB}$ activation in B-cell lymphoid malignancies. Semin Cancer Biol 39: 15-25, 2016.

44. Klemm S and Ruland J: Inflammatory signal transduction from the Fc epsilon RI to NF-kappa B. Immunobiology 211: 815-820, 2006.

45. Puri KD, Di Paolo JA and Gold MR: B-cell receptor signaling inhibitors for treatment of autoimmune inflammatory diseases and B-cell malignancies. Int Rev Immunol 32: 397-427, 2013.

46. Marmor MD, Skaria KB and Yarden Y: Signal transduction and oncogenesis by ErbB/HER receptors. Int J Radiat Oncol Biol Phys 58: 903-913, 2004

47. Hoeben A, Landuyt B, Highley MS, Wildiers H, Van Oosterom AT and De Bruijn EA: Vascular endothelial growth factor and angiogenesis. Pharmacol Rev 56: 549-580, 2004.

48. Harrison GS, Wierman ME, Nett TM and Glode LM: Gonadotropin-releasing hormone and its receptor in normal and malignant cells. Endocr Relat Cancer 11: 725-748, 2004.

49. Liu X and Li N: Regularity analysis on clinical treatment in primary liver cancer by traditional Chinese medicine. Zhongguo Zhong Yao Za Zhi 37: 1327-1331, 2012 (In Chinese).

50. Zhou X, Menche J, Barabási AL and Sharma A: Human symptoms-disease network. Nat Commun 5: 4212, 2014

51. Goh KI, Cusick ME, Valle D, Childs B, Vidal M and Barabási AL: The human disease network. Proc Natl Acad Sci USA 104: 8685-8690, 2007.

52. Liu AL and Du GH: Network pharmacology: New guidelines for drug discovery. Yao Xue Xue Bao 45: 1472-1477, 2010.

53. Wang Y, Fan X, Qu H, Gao X and Cheng Y: Strategies and techniques for multi-component drug design from medicinal herbs and traditional Chinese medicine. Curr Top Med Chem 12: 1356-1362, 2012.

54. Cai SQ, Wang X, Shang MY, Xu F and Liu GX: 'Efficacy Theory' may help to explain characteristic advantages of traditional Chinese medicines. Zhongguo Zhong Yao Za Zhi 40: 3435-3443, 2015 (In Chinese). 